\title{
Some Properties of Preposition and Subordinate Conjunction
} Attachments*

\author{
Alexander S. Yeh and Marc B. Vilain \\ MITRE Corporation \\ 202 Burlington Road \\ Bedford, MA 01730 \\ USA \\ $\{$ asy,mbv\}@mitre.org \\ phone\# +1-781-271-2658
}

\begin{abstract}
Determining the attachments of prepositions and subordinate conjunctions is a key problem in parsing natural language. This paper presents a trainable approach to making these attachments through transformation sequences and error-driven learning. Our approach is broad coverage, and accounts for roughly three times the attachment cases that have previously been handled by corpus-based techniques. In addition, our approach is based on a simplified model of syntax that is more consistent with the practice in current state-of-the-art language processing systems. This paper sketches syntactic and algorithmic details, and presents experimental results on data sets derived from the Penn Treebank. We obtain an attachment accuracy of $75.4 \%$ for the general case, the first such corpus-based result to be reported. For the restricted cases previously studied with corpusbased methods, our approach yields an accuracy comparable to current work (83.1\%).
\end{abstract}

\section{Introduction}

Determining the attachments of prepositions and subordinate conjunctions is an important problem in parsing natural language. It is also an old problem that continues to elude a complete solution. A classic example of the problem is the sentence "I saw a man with a telescope", where who had the telescope is ambiguous.

Recently, the preposition attachment problem has been addressed using corpus-based methods (Hindle and Rooth, 1993; Ratnaparkhi

\footnotetext{
- This paper reports on work performed at the MITRE Corporation under the support of the MITRE Sponsored Research Program. Useful advice was provided by Lynette Hirschman and David Palmer. The experiments made use of Morgan Pecelli's noun/verb group annotations and some of David Day's programs.
}

et al., 1994; Brill and Resnik, 1994; Collins and Brooks, 1995; Merlo et al., 1997). The present paper follows in the path set by these authors, but extends their work in significant ways. We made these extensions to solve this problem in a way that can be directly applied in running systems in such application areas as information extraction or conversational interfaces.

In particular, we have sought to produce an attachment decision procedure with far broader coverage than in earlier approaches. Most research to date has focussed on a subset of the attachment problem that only covers $25 \%$ of the problem instances in our training data, the socalled binary VNP subset. Even the broader $\mathrm{V}[\mathrm{NP}]^{*}$ subset addressed by (Merlo et al., 1997) only accounts for $33 \%$ of the problem instances. In contrast, our approach attempts to form attachments for as much as $89 \%$ of the problem instances (modulo some cases that are either pathological or accounted for by other means).

Work to date has also been concerned primarily with reproducing the structure of Treebank annotations. In other words, the underlying syntactic paradigm has been the traditional notion of full sentential parsing. This approach differs from the parsing models currently being explored by both theorists and practitioners, which include semi-parsing strategies and finitestate approximations to context-free grammars.

Our approach to syntax uses a cascade of rule sequence processors, each of which can be thought of as approximating some aspect of the underlying grammar by finite-state transduction. We have thus had to extend previous work at the conceptual level as well, by recasting the preposition attachment problem in terms of the vocabulary of finite-state approximations (noun groups, etc.), rather than the traditional syntactic categories (noun phrases, etc.). 
Much of the present paper is thus concerned with describing our extensions to the preposition attachment problem. We present the problem scope of interest to us, as well as the data annotations required to support our investigation. We also present a decision procedure for attaching prepositions and subordinate conjunctions. The procedure is trained through error-driven transformation learning (Brill, 1993), and we present a number of training experiments and report on the performance of the trained procedure. In brief, on the restricted VNP problem, our procedure achieves nearly the same level of test-set performance (83.1\%) as current state-of-the-art systems (84.5\% (Collins and Brooks, 1995)). On the unrestricted data set, our procedure achieves an attachment accuracy of $75.4 \%$.

\section{Syntactic Considerations}

Our outlook on the attachment problem is influenced by our approach to syntax, which simplifies the traditional parsing problem in several ways. As with many approaches to processing unrestricted text, we do not attempt as a primary goal to derive spanning sentential parses. Instead, we approximate spanning parses through successive stages of partial parsing. For the purpose of the present paper, we need to mostly be concerned with the level of analysis of core noun phrases and verb phrases. By core phrases, we mean the kind of nonrecursive simplifications of the NP and VP that in the literature go by names such as noun/verb groups (Appelt et al., 1993) or chunks, and base NPs (Ramshaw and Marcus, 1995).

The common thread between these approaches and ours is to approximate full noun phrases or verb phrases by only parsing their non-recursive core, and thus not attaching modifiers or arguments. For English noun phrases, this amounts to roughly the span between the determiner and the head noun; for English verb phrases, the span runs roughly from the auxiliary to the head verb. We call such simplified syntactic categories groups, and consider in particular noun, verb, adverb and adjective groups.

For noun groups in particular, the definition we have adopted also includes a limited number of constructs that encompass some depthbounded recursion. For example, we also in- clude in the scope of the noun group such complex determiners as partitives ("five of the suspects") and possessives ("John's book"). These constructs fall under the scope of our noun group model because they are easy to parse with simple finite-state cascades, and because they more intuitively match the notion of a core phrase than do their individual components. Our model of noun groups also includes an extension of the so-called named entities familiar to the information extraction community (Def, 1995). These consist of names of persons and organizations, location names, titles, dates, times, and various numeric expressions (such as money terms). Note in particular that titles and organization names often include embedded prepositional phrases (e.g., "Chief of Staff"). For such cases, as well as for partitives, we consider these embedded prepositional phrases to be within the noun group's scope, and as such are excluded from consideration as attachment problems. Also excluded are the auxiliary to's in verb groups for infinitives.

Once again, distinguishing syntax groups from traditional syntactic phrases (such as NPs) is of interest because it singles out what is usually thought of as easy to parse, and allows that piece of the parsing problem to be addressed by such comparatively simple means as finite-state machines or transformation sequences. What is then left of the parsing problem is the difficult stuff: namely the attachment of prepositional phrases, relative clauses, and other constructs that serve in modificational, adjunctive, or argument-passing roles. This part of the problem is harder both because of the ambiguous attachment location, and because the right combination of knowledge required to reduce this ambiguity is elusive.

\section{The Attachment Problem}

Given these syntactic preliminaries, we can now define attachment problems in terms of syntax groups. In addition to noun, verb, adjective and adverb groups, we also have I-groups. An I-group is a preposition (including multiple word prepositions) or subordinate conjunction (including wh-words and "that"). Once again prepositions that are embedded in such constructs as titles and names are not considered Igroups for our purposes. Each I-group in a sen- 
tence is viewed as attaching to one other group within that sentence. ${ }^{1}$ For example, the sentence "I had sent a cup to her." is viewed as

$$
[\mathrm{I}]_{n g}[\text { had sent }]_{v g, \triangleleft}[\mathrm{a} \text { cup }]_{n g}[\text { to }]_{I g, \triangleright}[\text { her }]_{n g} \text {. }
$$

where $\triangle$ indicates the attaching I-group and $\triangleleft$ indicates the group attached to.

Generally, coordinations of groups (e.g., dogs and cats) are left as separate groups. However, prenominal coordination (e.g. dog and cat food) is deemed as one large noun group.

Attachments not to try: Our system is designed to attach each I-group in a sentence to one other group in the sentence on that Igroup's left. In our sample data, about $11 \%$ of the I-groups have no left ambiguity (either no group on the left to attach to or only 1 group). A few (less than $0.5 \%$ ) of the I-groups have no group to its right. All of these I-groups count as attachments not handled by our system and our system does not attempt to resolve them.

Attachments to try: The rest of the I-groups each have at least 2 groups on their left and 1 group on their right from the I-group's sentence, and these are the I-groups that our system tries to handle ( $89 \%$ of all the problems in the data).

\section{Properties of Attachments to Try}

In order to understand how our technique handles the attachments that follow this pattern, it is helpful to consider the properties of this class of attachments. What we detail here is a specific analysis of our test data (called $7 \times 9 \times$ ). Our training sample is similar.

In $7 \times 9 x, 2.4 \%$ of the attachments turn out to be of a form that guarantees our system will fail to resolve them. $83 \%$ of these unresolvable "attachments" are about evenly divided between right attachments and left attachments to a coordination of groups (which in our framework is split into 2 or more groups). A right attachment example is that "at" attaches to "lost" in "that at home, they lost a key." A coordination attachment example is "with" attaching to the coordination "cats and dogs" in "cats and dogs with tags". The other $17 \%$ were either lexemes erroneously tagged as prepositions/subordinate conjunctions or past participles, or were $w h$-words that are actually part

\footnotetext{
${ }^{1}$ Sentential level attachments are deemed to be to the main verb in the sentence attached to.
}

of a question (and not acting as a subordinate conjunction).

In $7 x 9 x, 67.7 \%$ of attachments are to the adjacent group on the I-group's immediate left. Our system uses as a starting point the guess that all attachments are to the adjacent group.

The second most likely attachment point is the nearest verb group to the I-group's left. A surprising $90.3 \%$ of the attachments are to either this verb group or to the adjacent group. ${ }^{2}$ In our experiments, limiting the choice of possible attachment points to these two tended to improve the results and also increased the training speed, the latter often by a factor of 3 to 4 .

Neither of these percentages include attachments to coordinations of groups on the left, which are unhandleable. Including these attachments would add $\sim 1 \%$ to each figure.

The attachments can be divided into six categories, based on the contents of the I-group being attached and the types of groups surrounding that I-group. The categories are:

vnpn The I-group contains a preposition. Next to the preposition on both the left and the right are noun groups. Next to the left noun group is a verb group. A member of this category is the $\left[\right.$ to $_{I_{g}}$ in the sentence "[I] $]_{n g}[\text { had sent }]_{v g}$ [a cup $]_{n g}[\text { to }]_{I g}[\text { her }]_{n g} . "$

vnp̄ Like vnpn, but next to the preposition on the right is not a noun group.

vinpn Like vnpn, but the left neighbor of the left noun group is not a verb group.

$\overline{\mathbf{v}} \mathbf{n p} \overline{\mathbf{n}}$ Another variation on vnpn.

$\mathbf{x} \overline{\mathbf{n}} \mathbf{x}$ The I-group contains a preposition. But its left neighbor is not a noun group. The $\mathbf{x}$ 's stand for groups that need to exist, but can be of any type.

xxsx The I-group has a subordinate conjunction (e.g. which) instead of a preposition. ${ }^{3}$

Table 1 shows how likely the attachments in $7 \times 9 \times$ that belong to each category are

- to attach to the left adjacent group $(A)$

\footnotetext{
${ }^{2}$ This attachment preference also appears in the large data set used in (Merlo et al., 1997).

${ }^{3} \mathrm{~A}$ word is deemed a preposition if it is among the 66 prepositions listed in Section 6.2's lt data set. Unlisted words are deemed subordinate conjunctions.
} 
- to attach to either the left adjacent group or the nearest verb group on the left $(V-A)$

- to have an attachment that our system actually cannot correctly handle (Err).

The table also gives the percentage of the attachments in $7 \times 9 \times$ that belong in each category (Prevalence). The $A$ and $V-A$ columns do not include attachments to coordinations of groups.

\begin{tabular}{|c|c|c|c|c|}
\hline Category & A & $\overline{V-A}$ & Err & Prev \\
\hline vnpn & $55.6 \%$ & $97.3 \%$ & $0.8 \%$ & $22.8 \%$ \\
\hline vnpn & $44.4 \%$ & $92.6 \%$ & $0.0 \%$ & $2.4 \%$ \\
\hline$\overline{\mathbf{v}} \mathbf{n p n}$ & $61.4 \%$ & $85.1 \%$ & $2.5 \%$ & $30.7 \%$ \\
\hline$\overline{\mathbf{v}} \mathbf{n p \overline { \mathbf { n } }}$ & $37.7 \%$ & $83.0 \%$ & $3.8 \%$ & $2.4 \%$ \\
\hline $\mathbf{x} \overline{\mathbf{n}} \mathbf{p x}$ & $85.6 \%$ & $93.6 \%$ & $3.3 \%$ & $28.3 \%$ \\
\hline xxsx & $74.3 \%$ & $84.2 \%$ & $3.3 \%$ & $13.4 \%$ \\
\hline Overall & $67.7 \%$ & $90.3 \%$ & $2.4 \%$ & $100 \%$ \\
\hline
\end{tabular}

Table 1: Category properties in $7 \times 9 x$

Much of the corpus-based work on attaching prepositions (Ratnaparkhi et al., 1994; Brill and Resnik, 1994; Collins and Brooks, 1995) has dealt with the subset of category vnpn problems where the preposition actually attaches to either the nearest verb or noun group on the left. Some earlier work (Hindle and Rooth, 1993) also handled the subset of vnpñ category problems where the attachment is either to the nearest verb or noun group on the left.

Some later work (Merlo et al., 1997) dealt with handling from 1 to 3 prepositional phrases in a sentence. The work dealt with prepositions in "group" sequences of VNP, VNPNP and VNPNPNP, where the prepositions attach to one of the mentioned noun or verb groups (as opposed to an earlier group on the left). So this work handles attachments that can be found in

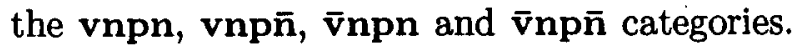
Still, this work handles less than an estimated $33 \%$ of our sample text's attachments. ${ }^{4}$

\footnotetext{
(Merlo et al., 1997) searches the Penn Treebank for data samples that they can handle. They find phrases where $78 \%$ of the items to attach belong to either the vnpn or vnpñ categories. So in Penn Treebank, they handle 1.28 times more attachments than the other work mentioned in this paper. This other work handles less than $25 \%$ of the attachments in our sample data.
}

\section{Processing Model}

Our attachment system is an extension of the rule-based system for VNPN binary prepositional phrase attachment described in (Brill and Resnik, 1994). The system uses transformationbased error-driven learning to automatically learn rules from training examples.

One first runs the system on a training set, which starts by guessing that each I-group attaches to its left adjacent group. This training run moves in iterations, with each iteration producing the next rule that repairs the most remaining attachment errors in the training set. The training run ends when the next rule found repairs less than a threshold number of errors.

The rules are then run in the same order on the test set (which also starts at an all adjacent attachment state) to see how well they do.

The system makes its decisions based on the head (main) word of each of the groups examined. Like the original system, our system can look at the head-word itself and also all the semantic classes the head-word can belong to. The classes come from Wordnet (Miller, 1990) and consist of about 25 noun classes (e.g., person, process) and 15 verb classes (e.g., change, communication, status). As an extension, our system also looks at the word's partof-speech, possible stem(s) and possible subcategorization/complement categories. The latter consist of over 100 categories for nouns, adjectives and verbs (mainly the latter) from Comlex (Wolff et al., 1995). Example categories include intransitive verbs and verbs that take 2 prepositional phrases as a complement (e.g., fly in "I fly from here to there."). In addition, Comlex gives our system the possible prepositions (e.g. from and to for the verb $f(y)$ and particles used in the possible subcategorizations.

The original system chose between two possible attachment points, a verb and a noun. Each rule either attempted to move left (attach to the verb) or move right (attach to the noun). Our extensions include as possible attachment points every group that precedes the attaching I-group and is in the I-group's sentence. The rules now can move the attachment either left or right from the current guess to the nearest group that matches the rule's constraints.

In addition to running the training and test with ALL possible attachment points (every 
preceding group) available, one can also restrict the possible attachment points to only the group Adjacent to the I-group and the nearest Verb group on the left, if any ( $\mathbf{V}-\mathbf{A})$. One uses the same attachment choice (ALL versus $\mathbf{V}$-A) in the training run and corresponding test run.

\section{Experiments}

\subsection{Data preparation}

Our experiments were conducted with data made available through the Penn Treebank annotation effort (Marcus et al., 1993). However, since our grammar model is based on syntax groups, not conventional categories, we needed to extend the Treebank annotations to include the constructs of interest to us.

This was accomplished in several steps. First, noun groups and verb groups were manually annotated using Treebank data that had been stripped of all phrase structure markup. ${ }^{5}$ This syntax group markup was then reconciled with the Treebank annotations by a semi-automatic procedure. Usually, the procedure just needs to overlay the syntax group markup on top of the Treebank annotations. However, the Treebank annotations often had to be adjusted to make them consistent with the syntax groups (e.g., verbal auxiliaries need to be included in the relevant verb phrase). Some $4-5 \%$ of all Treebank sentences could not be automatically reconciled in this way, and were removed from the data sets for these experiments.

The reconciliation procedure also automatically tags the data for part-of-speech, using a high-performance tagger based on (Brill, 1993). Finally, the reconciler introduces adjective, adverb, and I-group markup. I-groups are created for all lexemes tagged with the IN, TO, WDT, WP, WP\$ or WRB parts of speech, as well as multi-word prepositions such as according to.

The reconciled data are then compiled into attachment problems using another semiautomatic pattern-matching procedure. $8 \%$ of the cases did not fit into the patterns and required manual intervention.

We split our data into a training set (files 2000,2013 , and 200-269) and a test set (files 270-299). Because manual intervention is time consuming, it was only performed on the test set. The training set (called 0x6x) has 2615

\footnotetext{
${ }^{5}$ We used files 200-299, along with files 2000 and 2013.
}

attachment problems and the test set (called $7 \times 9 x)$ has 2252 attachment problems.

\subsection{Preliminary test}

The preliminary experiment with our system compares it to previous work (Ratnaparkhi et al., 1994; Brill and Resnik, 1994; Collins and Brooks, 1995) when handling VNPN binary PP attachment ambiguity. In our terms, the task is to determine the attachment of certain vnpn category I-groups. The data originally was used in (Ratnaparkhi et al., 1994) and was derived from the Penn Treebank Wall St. Journal. It consists of about 21,000 training examples (call this $l t$, short for large-training) and about 3000 test examples. The format of this data is slightly different than for $0 \times 6 x$ and $7 \times 9 x$ : for each sample, only the 4 mentioned groups (VNPN) are provided, and for each group, this data just provides the head-word. As a result, our part-of-speech tagger could not run on this data, so we temporarily adjusted our system to only consider two part-of-speech categories: numbers for words with just commas, periods and digits, and non-numbers for all other words. The training used a 3 improvement threshold. With these rules, the percent correct on the test set went from $59.0 \%$ (guess all adjacent attachments) to $83.1 \%$, an error reduction of $58.9 \%$. This result is just a little behind the current best result of $84.5 \%$ (Collins and Brooks, 1995) (using a binomial distribution test, the difference is statistically significant at the $2 \%$ level). (Collins and Brooks, 1995) also reports a result of $81.9 \%$ for a word only version of the system (Brill and Resnik, 1994) that we extend (difference with our result is statistically significant at the $4 \%$ level). So our system is competitive on a known task.

\subsection{The main experiments}

We made 4 training and test run pairs:

\begin{tabular}{|c|c||c|r|r|}
\hline TR SET & AP & RULES & COR & ER \\
\hline \hline $0 \times 6 \times$ & ALL & 159 & $70.1 \%$ & $7.4 \%$ \\
\hline $0 \times 6 \times$ & V-A & 118 & $73.0 \%$ & $16.3 \%$ \\
\hline$l t^{-}$ & V-A & 444 & $75.4 \%$ & $24.0 \%$ \\
\hline$l t 2^{-}$ & V-A & 451 & $74.7 \%$ & $21.8 \%$ \\
\hline
\end{tabular}

The test set was always $7 \times 9 \times$, which starts at $67.7 \%$ correct. The results report the number of RULES the training run produces, as well 
as the percent CORrect and Error Reduction in the test. One source of variation is whether ALL or the V-A Attachment Points are used. The other source is the TRaining SET used.

The set $l t^{-}$is the set $l t$ (Section 6.2) with the entries from Penn Treebank Wall St. Journal files 270 to 299 (the files used to form the test set) removed. About 600 entries were removed. Several adjustments were made when using $l t^{-}$: The part-of-speech treatment in Section 6.2 was used. Because $l t^{-}$only gives two possible attachment points (the adjacent noun and the nearest verb), only $\mathbf{V}-\mathbf{A}$ attachment points were used. Finally, because $l t^{-}$is much slower to train on than $0 \times 6 x$, training used a 3 improvement threshold. For 0x6x, a 2 improvement threshold was used.

Set $l t 2$ is the data used in (Merlo et al., 1997) and has about 26000 entries. The set $l t 2^{-}$is the set $l t 2$ with the entries from Penn Treebank files 270-299 removed. Again, about 600 entries were removed. Generally, lt2 has no information on the word(s) to the right of the preposition being attached, so this field was ignored in both training and test. In addition, for similar reasons as given for $\mathrm{lt}^{-}$, the adjustments made when using $l t^{-}$were also made when using $l t 2^{-}$.

If one removes the $l t 2^{-}$results, then all the COR results are statistically significantly different from the starting $67.7 \%$ score and from each other at a $1 \%$ level or better. In addition, the $l t 2^{-}$and $l t^{-}$results are not statistically significantly different (even at the $20 \%$ level).

$l t 2^{-}$has more data points and more categories of data than $l t^{-}$, but the $l t^{-}$run has the best overall score. Besides pure chance, two other possible reasons for this somewhat surprising result are that the $l t 2^{-}$entries have no information on the word(s) to the right of the preposition being attached ( $l t^{-}$does) and both datasets contain entries not in the other dataset.

When looking at the $l t^{-}$run's remaining errors, $43 \%$ of the errors were in category $\overline{\mathbf{v}} \mathbf{n p n}$, $21 \%$ in vnpn, $16 \%$ in $\mathbf{x} \overline{\mathbf{n} x}, 13 \%$ in xxsx, $4 \%$ in $\overline{\mathbf{v}} \mathbf{n p} \overline{\mathbf{n}}$ and $3 \%$ in vnp̄

\subsection{Afterwards}

The $l^{-}$run has the best overall score. However, the $l t^{-}$run does not always produce the best score for each category. Below are the scores (number correct) for each run that has a best score (bold face) for some category:

\begin{tabular}{|c|c|c|c|}
\hline Category & $0 \times 6 \times(V-A)$ & $l t^{-}$ & $l t 2^{-}$ \\
\hline vnpn & $\overline{\overline{345}}$ & $\overline{\mathbf{3 9 7}}$ & $\overline{\overline{374}}$ \\
\hline vnpñ & $\overline{35}$ & 39 & 34 \\
\hline$\overline{\mathbf{v} n p n}$ & 441 & 454 & 458 \\
\hline$\overline{\mathbf{v}} \mathbf{n p \overline { n }}$ & $\overline{32}$ & 29 & 36 \\
\hline $\mathbf{x \overline { \mathbf { n } } \mathbf { p x }}$ & 554 & 551 & 557 \\
\hline $\operatorname{xxsx}$ & 236 & 229 & 224 \\
\hline
\end{tabular}

The location of most of the best subscores is not surprising. Of the training sets, $l t^{-}$has the most vnpn entries, ${ }^{6} l t 2^{-}$has the most $\overline{\mathbf{v}}$ ptype entries and $0 \times 6 \times$ has the most xxsx entries. The best vnp̄̄ and $\mathbf{x} \overline{\mathbf{n}} \mathbf{p} \mathbf{x}$ subscore locations are somewhat surprising. The best vnpn̄ subscore is statistically significantly better than the $l t 2^{-}$ vnpn subscore at the $5 \%$ level. A possible explanation is that the vnp $\bar{n}$ and vnpn categories are closely related. The best $\mathbf{x} \overline{\mathbf{n}} \mathbf{p} \mathbf{x}$ subscore is not statistically significantly better than the $l t^{-}$ xn̄px subscore, even at the $25 \%$ level. Besides pure chance, a possible explanation is that the $\mathbf{x n p x}$ category is related to the four np-type categories (where $l t 2^{-}$has the most entries).

The fact that the subscores for the various categories differ according to training regimen suggests a system architecture that would exploit this. In particular, we might apply different rule sets for each attachment category, with each rule set trained in the optimal configuration for that category. We would thus expect the overall accuracy of the attachment procedure to improve overall. To estimate the magnitude of this improvement, we calculated a post-hoc composite score on our test set by combining the best subscore for each of the 6 categories. When viewed as trying to improve upon the $l t^{-}$subscores, the new $\overline{\mathbf{v}} \mathbf{n p} \overline{\mathbf{n}}$ subscore is statistically significantly better ( $4 \%$ level) and the new xxsx subscore is mildly statistically significantly better ( $20 \%$ level). The new v̄npn and $\mathbf{x} \bar{n} \mathbf{p} \mathbf{x}$ subscores are not statistically significantly better, even at the $25 \%$ level. This combination yields a post-hoc improved score of $76.5 \%$. This is of course only a post-hoc estimate, and we would need to run a new independent test to verify the actual validity of this effect. Also, this estimate is only mildly statistically significantly better ( $13 \%$ level) than the existing $75.4 \%$ score.

\footnotetext{
${ }^{6}$ For vnpn, the $l t^{-}$score is statistically significantly better than the $l t 2^{-}$score at the $2 \%$ level.
} 


\section{Discussion}

This paper presents a system for attaching prepositions and subordinate conjunctions that just relies on easy-to-find constructs like noun groups to determine when it is applicable. In sample text, we find that the system is applicable for trying to attach $89 \%$ of the prepositions/subordinate conjunctions that are outside of the easy-to-find constructs and is $75.4 \%$ correct on the attachments that it tries to handle. In this sample, we also notice that these attachments very much tend to be to only one or two different spots and that the attachment problems can be divided into 6 categories. One just needs those easy-to-find constructs to determine the category of an attachment problem.

The $75.4 \%$ results may seen low compared to parsing results like the $88 \%$ precision and recall in (Collins, 1997), but those parsing results include many easier-to-parse constructs. (Manning and Carpenter, 1997) presents the VNPN example phrase "saw the man with a telescope", where attaching the preposition incorrectly can still result in $80 \%$ (4 of 5) recall, $100 \%$ precision and no crossing brackets. Of the 4 recalled constructs, 3 are easy-to-parse: 2 correspond to noun groups and 1 is the parse top level.

In our experiments, we found that limiting the choice of possible attachment points to the two most likely ones improved performance. This limiting also lets us use the large training sets $l t^{-}$and $l t 2^{-}$. In addition, we found that different training data produces rules that work better in different categories. This latter result suggests trying a system architecture where each attachment category is handled by the rule set most suited for that category.

In the best overall result, nearly half of the remaining errors occur in one category, vnpn, so this is the category in need of most work.

Another topic to examine is how many of the remaining attachment errors actually matter. For instance, when one's interest is on finding a semantic interpretation of the sentence "They flash letters on a screen.", whether on attaches to flash or to letters is irrelevant. Both the letters are, and the flashing occurs, on a screen.

\section{References}

D. Appelt, J. Hobbs, J. Bear, D. Israel, and M. Tyson. 1993. Fastus: A finite-state pro- cessor for information extraction. In 13th Intl. Conf. On Artificial Intelligence (IJCAI).

E. Brill and P. Resnik. 1994. A rule-based approach to prepositional phrase attachment disambiguation. In 15th Intermational Conf. on Computational Linguistics (COLING).

E. Brill. 1993. A Corpus-based Approach to Language Learning. Ph.D. thesis, U. Pennsylvania.

M. Collins and J. Brooks. 1995. Prepositional phrase attachment through a backedoff model. In Proc. of the 3rd Workshop on Very Large Corpora, Cambridge, MA, USA.

M. Collins. 1997. Three generative, lexicalized models for statistical parsing. In $A C L 97$.

Defense Advanced Research Projects Agency. 1995. Proc. 6th Message Understanding Conference (MUC-6), November.

D. Hindle and M. Rooth. 1993. Structural ambiguity and lexical relations. Computational Linguistics, 19(1):103-120.

C. Manning and B. Carpenter. 1997. Probabilistic parsing using left corner language models. In Proc. of the 5th Intl. Workshop on Parsing Technologies.

M. Marcus, B. Santorini, and M. Marcinkiewicz. 1993. Building a large annotated corpus of english: the penn treebank. Computational Linguistics, 19(2).

P. Merlo, M. Crocker, and C. Berthouzoz. 1997. Attaching multiple prepositional phrases: Generalized backed-off estimation. In Proc. of the 2nd Conf. on Empirical Methods in Natural Language Processing. ACL.

G. Miller. 1990. Wordnet: an on-line lexical database. Intl. J. of Lexicography, 3(4).

L. Ramshaw and M. Marcus. 1995. Text chunking using transformation-based learning. In Proc. 3rd Workshop on Very Lange Corpora.

A. Ratnaparkhi, J. Reynar, and S. Roukos. 1994. A maximum entropy model for prepositional phrase attachment. In Proc. of the Human Language Technology Workshop. Advanced Research Projects Agency, March.

S. Wolff, C. Macleod, and A. Meyers, 1995. Comlex Word Classes. C.S. Dept., New York U., Feb. prepared for the Linguistic Data Consortium, U. Pennsylvania. 